\title{
Original
}

\section{Transgenic mouse model exhibiting weak red fluorescence before and strong green fluorescence after Cre/loxP-mediated recombination}

\author{
Ryoko AKAI ${ }^{1)}$, Michiko SAITO ${ }^{2)}$, Kenji $\mathrm{KOHNO}^{3)}$ and Takao IWAWAKI ${ }^{1)}$ \\ 1)Division of Cell Medicine, Department of Life Science, Medical Research Institute, Kanazawa Medical University, 1-1 \\ Daigaku, Uchinada, Kahoku, Ishikawa 920-0293, Japan \\ ${ }^{2)}$ Bio-science Research Center, Kyoto Pharmaceutical University, 1 Shichono-cho, Misasagi, Yamashina, Kyoto 607-8412, \\ Japan \\ 3) Institute for Research Initiatives, Nara Institute of Science and Technology, 8916-5 Takayama, Ikoma, Nara 630-0192, Japan
}

\begin{abstract}
The Cre/loxP system is an indispensable tool for temporal and spatial control of gene function in mice. Many mice that express Cre and carry loxP sites in their genomes have been bred for functional analysis of various genes in vivo. Also, several reporter mice have been generated for monitoring of recombination by the Cre/loxP system. We have developed a Cre reporter gene with DsRed1 and Venus that exhibits a strong red fluorescence before and a strong green fluorescence after Cre/loxP-mediated recombination in experiments using NIH3T3 cells. However, a transgenic mouse introduced with the same reporter gene exhibits a weak red fluorescence before and a strong green fluorescence after Cre/loxP-mediated recombination. This property manifested ubiquitously in this mouse model and was maintained stably in mouse-derived fibroblasts. Use of the mouse model exhibiting the stronger red fluorescence might result in confusion of the Cre-dependent signal with false signals, because the Venus signal includes some fluorescence in the red region of the spectrum and the DsRed1 signal includes some fluorescence in the green region. However, we fortuitously obtained reporter mice that exhibit a weaker red fluorescence before Cre/loxP-mediated recombination. The use of this mouse model would decrease concern regarding errors in the identification of signals and should increase certainty in the detection of Cre activity in vivo. Key words: Cre/loxP, green fluorescent protein (GFP), model mouse, red fluorescent protein (RFP), reporter
\end{abstract}

\section{Introduction}

Cre recombinase (Cre), which is derived from P1 bacteriophage, is a $38 \mathrm{kDa}$ protein composed of four subunits that recognizes pairs of specific 34 bp DNA sequences called loxP sites $[3,6]$, and induces accurate excision, inversion, insertion, and translocation of genomic DNA, depending on the position of these loxP sites [16]. The Cre/loxP system was first used in yeast and in cultured mammalian cells $[18,19]$ and it has recently become an indispensable tool for temporal and spatial control of gene function in mice [10]. Consequently, many scientists have generated various mice that express Cre and carry loxP sites in the murine genome. For example, some research groups have developed mouse lines with an insulin-promoter-driven $\mathrm{Cre}$ transgene to control the expression of a specific gene in pancreatic $\beta$ cells [12]. Other groups have established mouse lines with exon(s) of the Ern1 gene flanked by lox $P$ sites to examine the effects of deletion of the gene in vivo $[8,20,23]$. In addition, several reporter mice have been used to monitor recombination by the Cre/ loxP system. Some of these mice have an exogenous lac $Z$ gene as a reporter that is transcriptionally activated in a Cre/loxP-dependent manner [1,21]. Others have two fluorescent reporter genes that are alternatively ex-

(Received 4 July 2019 / Accepted 26 January 2020 / Published online in J-STAGE 1 March 2020)

Corresponding author: T. Iwawaki.e-mail: iwawaki@kanazawa-med.ac.jp

Supplementary Figures: refer to J-STAGE: https://www.jstage.jst.go.jp/browse/expanim

This is an open-access article distributed under the terms of the Creative Commons Attribution Non-Commercial No Derivatives (by-nc-nd) License <http://creativecommons.org/licenses/by-nc-nd/4.0/>.

C2020 Japanese Association for Laboratory Animal Science 
pressed before and after Cre/loxP-mediated recombination $[4,5]$. In the former case, because mouse or tissue samples have to be treated by paraformaldehyde fixation and X-gal staining [2], the Cre activity can be detected only in nonliving mice or nonliving tissues, whereas on the latter case, because the mice only require excitation with light of specific wavelength [7], the Cre activity can be detected in living mice or living tissues.

These Cre reporter mice are usually designed to express variants of jellyfish-derived green fluorescent protein (GFP) or reef coral-derived red fluorescent protein (RFP). Thus, Cre activity can be easily detected from a change in the color of the fluorescence from red to green or from green to red [4,5]. However, it is possible that the Cre-dependent signal from a mouse model exhibiting strong fluorescence before Cre/loxP-mediated recombination might be confused with false signals, because the GFP signal includes some fluorescence in the red region of the spectrum, and the RFP signal includes some fluorescence in the green region of the spectrum [14]. Here we report our fortuitous production of a transgenic mouse model that exhibits a weak red fluorescence before Cre/loxP-mediated recombination and strong green fluorescence afterwards. This property is manifested ubiquitously in this mouse model. The use of this model should decrease concern about errors in the judgment of signals and might increase certainty in respect of the detection of Cre activity in vivo.

\section{Materials and Methods}

\section{Gene constructs}

pCAX-CREI-RtoG was made by insertion of a VenusFlag fragment and a DsRed1-HA fragment into the KpnI/ Xhol sites and the EcoRI/BamHI sites of pCAX-MCSloxP-1, respectively. pCAX-MCS-loxP-1 was made by insertion of a loxP-int/pA-loxP fragment into the HindIII/BamHI sites of pCAX. The Venus-Flag fragment was produced by PCR using 5' -ccg gaa ttc cca cca tgg tga gca agg gcg agg agc tgt tca cc-3' as the forward primer, 5'-cgc gga tcc aag ctt tta ctt gtc atc gtc gtc ctt gta gtc ctt gta cag ctc gtc cat gc-3' as the reverse primer, and Venus full-length cDNA as the template. The Venus full-length cDNA was gifted from Dr. Takeharu Nagai (Osaka University). The DsRed1-HA fragment was produced by PCR using 5' -cgg ggt acc cca cca tgg tge get cct cca aga acg tca tca agg-3' as the forward primer, 5 '-ccg ctc gag aag ctt cta agc gta atc tgg aac atc gta tgg gta cag gaa cag gtg gtg gcg gcc ctc g-3' as the reverse primer, and DsRed1 full-length cDNA as the template. The DsRed1 full-length cDNA was obtained commercially from Clontech Laboratories Inc. The loxP-int/
pA-loxP fragment was produced by PCR using 5'-ccc aag ctt ata act tcg tat agc ata cat tat acg aag tta tcg gta cca gat atc tge agc tcg agg cta get agg tag cta gag g-3, as the forward primer, 5' -cgc gga tcc atc gat gcg gcc gcg aat tcg ata act tcg tat aat gta tgc tat acg aag tta ttc gec cat ctt ctg aag ctg atc c-3' as the reverse primer, and the intron and polyadenylation site region derived from the SV40 T antigen gene on pCAX as the template.

pCAX-Cre was made by insertion of a Cre fragment into the XhoI/BamHI sites of pCAX2. The Cre fragment was produced by PCR using 5'-ccg ctc gag gga tcc cca cca tgg cac cca aga aga aga gg-3' as the forward primer, 5'-gga aga tct cta atc gcc atc ttc cag cag g-3' as the reverse primer, and Cre full-length cDNA as the template. The Cre full-length cDNA was obtained commercially from Gene Bridges.

\section{Transgenic mice}

The 5.3-kb SpeI-SfiI fragment of pCAX-CREI-RtoG was microinjected as a transgene into fertilized mouse eggs (C57BL/6JJcl) to develop the CREI mouse line, and genotyping of the offspring was performed by PCR using the following primers: 5'-cgg cca cga gtt cga gat cg-3' and 5' -tgt aga tgg act tga act cc-3'.

Meox-Cre mice (Stock number: 003755) were obtained from The Jackson Laboratory. Genotyping of the offspring was performed by PCR using the following primers: 5' -ggg acc acc ttc ttt tgg ctt c-3', 5'-aag atg tgg aga gtt cgg ggt ag-3', and 5' -cca gat cet cet cag aaa tca gc-3'.

RIP-Cre mice (Stock number: 003573) were obtained from The Jackson Laboratory. Genotyping of the offspring was performed by PCR using the following primers: 5' -gcg gtc tgg cag taa aaa cta tc-3' and 5'-gtg aaa cag cat tgc tgt cac tt-3'.

CREI mice, Meox-Cre, and RIP-Cre mice were maintained by mating with wild-type mice (C57BL/6JJcl). The protocols for the animal experiment were approved by the Animal Studies Committees at Nara Institute of Science and Technology (authorization number: 150) and at Kanazawa Medical University (authorization numbers: 2016-107 and 2017-51); all experiments were performed in accordance with the appropriate institutional guidelines.

\section{Southern blot analysis}

Genomic DNA was extracted from the tail tip of the mouse in accordance with a standard procedure. Aliquots of $10 \mu \mathrm{g}$ of genomic DNA were digested with HindIII, loaded into the appropriate lanes of $0.8 \%$ agarose gels, and transferred onto nylon membranes (\#60207; PALL, Port Washington, NY, USA). Hybridization was per- 
formed in $\mathrm{H}$ solution [500 $\mathrm{mM} \mathrm{Na}_{2} \mathrm{HPO}_{4}, 1 \mathrm{mM}$ ethylenediaminetetraacetic acid (EDTA), and 7\% sodium dodecylsulfate (SDS)] at $65^{\circ} \mathrm{C}$ for $16 \mathrm{~h}$. The membranes were washed four times with $\mathrm{W}$ solution $\left(40 \mathrm{mM} \mathrm{Na}_{2} \mathrm{H}-\right.$ $\mathrm{PO}_{4}$ and $1 \% \mathrm{SDS}$ ) at $65^{\circ} \mathrm{C}$ for $10 \mathrm{~min}$. To detect the CREI transgene, a ${ }^{32} \mathrm{P}$-labeled DsRed1 coding region was used as a probe.

\section{Northern blot analysis}

Total RNA was extracted from cultured cells and tissue fragments by using the Isogen reagent (\#311-02501; Nippon Gene, Tokyo, Japan). Aliquots of $5 \mu \mathrm{g}$ of total RNA were loaded into the appropriate lanes of $1 \%$ denaturing agarose gels and transferred onto nylon membranes (\#RPN303N; GE Healthcare, Little Chalfont, UK). Hybridization was performed in $\mathrm{H}$ solution (500 $\mathrm{mM} \mathrm{Na}_{2} \mathrm{HPO}_{4}, 1 \mathrm{mM}$ EDTA, and $7 \%$ SDS) at $65^{\circ} \mathrm{C}$ for $16 \mathrm{~h}$. The membranes were washed four times with $\mathrm{W}$ solution ( $40 \mathrm{mM} \mathrm{Na}_{2} \mathrm{HPO}_{4}$ and $1 \% \mathrm{SDS}$ ) at $65^{\circ} \mathrm{C}$ for 10 min. To examine the expression of DsRed1-HA mRNA and Venus-Flag mRNA, the ${ }^{32} \mathrm{P}$-labeled coding regions of DsRed1 and Venus, respectively, were used as probes.

\section{Western blot analysis}

The culture cells and the tissue fragments were lysed in SDS sample buffer [50 mM Tris-HCl, pH 6.8, 2\% SDS, $50 \mathrm{mM}$ dithiothreitol, $10 \%$ glycerol, and $1 \mathrm{mg} / \mathrm{ml}$ bromophenol blue]. The lysate was heated to $98^{\circ} \mathrm{C}$ for $10 \mathrm{~min}$, and SDS-PAGE was performed to separate the proteins in the lysate. After electrophoresis, the proteins were electrotransferred onto PVDF microporous membranes (\#IPVH00010; Merck, Darmstadt, Germany) and immunodetected with the anti-FLAG monoclonal antibody (\#F3165; Sigma-Aldrich, St. Louis, MO, USA), anti-HA monoclonal antibody (\#ab-hatag; Invivogen, San Diego, CA, USA), and anti-GAPDH monoclonal antibody (\#2118; Cell Signaling Technology, Beverly, MA, USA).

\section{Quantitative PCR analysis}

Quantitative PCR analysis was performed by using the TaqMan probe and Real-Time PCR systems (\#StepOnePlus; Thermo Fisher Scientific, Waltham, MA, USA). Three probe/primer sets (\#Mr04329676_mr, \#Mm00607939_s1 and \#4352339E; Thermo Fisher Scientific) were used to quantify the expression level of the Venus-Flag gene, the $\beta$-actin gene, and the Gapdh gene. The copy number of the CREI gene and the DsRed1-HA gene in genome and the expression level of the DsRed1HA gene were quantified by using 5 '-acg gcc acg agt tcg aga-3' as the forward primer, 5' -acg tac acc ttg gag ccg tac $t-3^{\prime}$ 'as the reverse primer, and 5'-FAM-aag ggc ggc ccc ct-MGB-3' as the probe. For the quantification of the gene expression level, mRNAs were previously reverse-transcribed by using the SuperScript first-strand synthesis system (\#11904-018; Thermo Fisher Scientific) in accordance with the manufacturer's instructions.

\section{Cell culture}

Mouse embryonic fibroblasts (MEFs) were isolated in accordance with a standard procedure [17]. Plasmid transfection into NIH3T3 cells was performed with an electroporator (\#4D-Nucleofector; Lonza, Basel, Switzerland) in accordance with the manufacturer's instructions. pCREI-RtoG, pCAX-Cre, and pCAX were used as plasmids for expression of the CREI gene, for the expression of $\mathrm{Cre}$, and for sham transfection, respectively. MEFs and transfected NIH3T3 cells were cultured in Dulbecco's modified Eagle's medium supplemented with $10 \%$ fetal bovine serum at $37^{\circ} \mathrm{C}$ under $5 \% \mathrm{CO}_{2}$. To inhibit proteasome activity, MEFs were treated with 20 $\mu \mathrm{M}$ MG132 (\#3175-v; Peptide Institute Inc., Osaka, Japan) for $6 \mathrm{~h}$.

\section{Isolation of islets from mouse pancreas}

Islets were isolated from pancreas of 8-week-old mice in accordance with a previous report [11].

\section{Imaging of fluorescent signals}

The embryos and placentas were collected surgically from the uteri of female mice at 12.5 days post coitum. The adult tissues were collected surgically from female mice at 5 or 6 weeks old. Fluorescent signals from them were imaged by using an in vivo imaging system (\#Lumina; Perkin Elmer, Waltham, MA, USA) or a fluorescence stereoscopic microscope (\#MVX10; Olympus, Tokyo, Japan) with a color charge-coupled device (CCD) camera (\#DP72; Olympus). Imaging of fluorescent signals from the MEFs and transfected NIH3T3 cells was performed by using a closed-box fluorescence microscope (\#FSX100; Olympus). The photographic conditions are indicated in the figures or in the figure legends.

\section{Flow cytometry analysis}

Preparation of cells were conducted in accordance with the manufacturer's protocol (https://www.bio-radantibodies.com/static/2015/flow-protocol/fc/fc1-preparation-of-cells.pdf). These cells were pre-treated with 20 $\mu$ M MG132 (\#3175-v; Peptide Institute Inc.) for $6 \mathrm{~h}$, and dissociated with nylon mesh (\#352340; Corning Inc., Corning, NY, USA) right before flow cytometry analysis. Fluorescent signals of cells were analyzed by using a flow cytometer (\#Accuri C6 Plus; Becton, Dickinson and Co., Franklin Lakes, NJ, USA). 


\section{Immunostaining of tissue sections}

All tissues were fixed with $4 \%$ paraformaldehyde and embedded in paraffin. The paraffin-embedded tissues were sliced in $2 \mu \mathrm{m}$ thick and placed on silane-coated slides. On each slide, DsRed1-HA and Venus-Flag were double immunostained with rabbit anti-RFP polyclonal antibody (\#ab62341; Abcam, Cambridge, UK ), goat anti-rabbit IgG polyclonal antibody conjugated with Alexa Fluor 594 (\#ab150080; Abcam), chicken anti-GFP polyclonal antibody (\#ab13970; Abcam), and goat antichicken IgY polyclonal antibody conjugated with Alexa Fluor 488 (\#ab150169; Abcam). Hoechst 33342 (\#53917191; Wako, Osaka, Japan) was used for counter staining of the tissues.

\section{Measurement of fluorescent signals}

Fluorescent signals from the embryos and placentas were measured with an in vivo imaging system (\#Lumina; Perkin Elmer), whereas those from MEFs and transfected NIH3T3 cells were measured with a fluorescence microplate reader (\#Fluoroskan; Thermo Fisher Scientific). Measurement conditions are indicated in the figures or in the figure legends.

\section{Results}

\section{Design of the fluorescent reporter for Cre/loxP-} mediated recombination

To detect Cre activity from changes in the fluorescence color from red to green, we constructed the reporter gene shown in Fig. 1A. The RFP and GFP are coded by the DsRed 1 gene and the Venus gene, respectively $[13,15]$. Each gene is connected to the intron and the polyadenylation site of a T-antigen gene derived from SV40. The two loxP sites are located before and after the DsRed1 gene. The HA-tag sequence and the FLAG-tag sequence are linked to the DsRed1 gene and to the Venus gene, respectively. Transcription of the DsRed1 gene or Venus gene is constitutively activated by the enhancer of the IE gene derived from CMV and the promoter of the $\beta$-actin gene derived from chick. This reporter construct

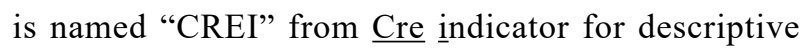
purposes here.

\section{Generation and characterization of CREI transgenic mice}

By microinjection of the CREI construct into fertilized mouse eggs, we generated a transgenic mouse (F0). Southern blot analysis and quantitative PCR analysis confirmed that the CREI mouse line had been introduced with ten copies of the transgene in the expected manner, and that the copy number of the transgene was sustained in subsequent generations of the CREI mouse line (Figs. $1 \mathrm{~B}$ and $\mathrm{C})$.

To examine their usefulness as Cre indicators, the CREI mice were mated with Meox-Cre mice that express Cre in the epiblasts restrictedly [22], and the mRNAs and proteins extracted from the offspring were examined by Northern blot analysis and Western blot analysis. Meox-Cre; CREI mice expectedly expressed DsRed1HA mRNA in all their tissues, and the Venus-Flag mRNA in all their tissues except for the placenta. On the other hand, the CREI (Meox-Cre ${ }^{-}$) mice also expressed DsRed1-HA mRNA in all tissues, but did not express Venus-Flag mRNA in any tissue. Wild-type (Meox-Cre-; $\left.\mathrm{CREI}^{-}\right)$mice and Meox-Cre $\left(\mathrm{CREI}^{-}\right)$mice did not express either the reporter mRNA in any tissue. DsRed1HA protein and Venus-Flag protein were expressed in the same manner as the respective mRNA (Fig. 2A). Quantitative PCR analysis additionally revealed that DsRed1-HA mRNA was expressed in similar levels in all tissues of both CREI mice and Meox-Cre; CREI mice, and that Venus-Flag mRNA was expressed in Meox-Cre; CREI mice at a similar level in all tissues except for the placenta. The same quantitative PCR analysis detected small amounts of Venus-Flag mRNA in all tissues of CREI mice and in the placenta of Meox-Cre; CREI mice. Neither reporter mRNA was detected in wild-type mice or in Meox-Cre mice (Fig. 2B). Furthermore the copy number of DsRed1-HA gene was compared between in genome of CREI (Meox-Cre ${ }^{-}$) mice and in that of MeoxCre; CREI mice. Quantitative PCR analysis confirmed that CREI (Meox-Cre ${ }^{-}$) mice expectedly had ten copies of DsRed1-HA gene in all examined tissues and MEFs, but that Meox-Cre; CREI mice had 6-8 copies of DsRed1-HA gene in all examined tissues and MEFs (Fig. 3).

Next, we examined the fluorescent signals of DsRed1HA and Venus-Flag from the embryo and the placenta of each mouse genotype. Observation with the fluorescence stereoscopic microscope and the color CCD camera showed that obvious Venus-Flag signals could be detected from the whole embryos of Meox-Cre; CREI mice, but not from any other embryos or placentas. On the other hand, DsRed1-HA signals were barely detected from either the placenta or embryo of any genotype of mouse (Fig. 4A). The fluorescent signals of Venus-Flag were consistent with the results from the expression analysis described above, whereas those of DsRed1-HA were inconsistent with those results. This inconsistency led us to hypothesize that the fluorescent signals of DsRed1-HA might be markedly weaker in the CREI mouse line. Thus, fluorescent signals of DsRed1HA and Venus-Flag were recorded by using an in vivo 
A
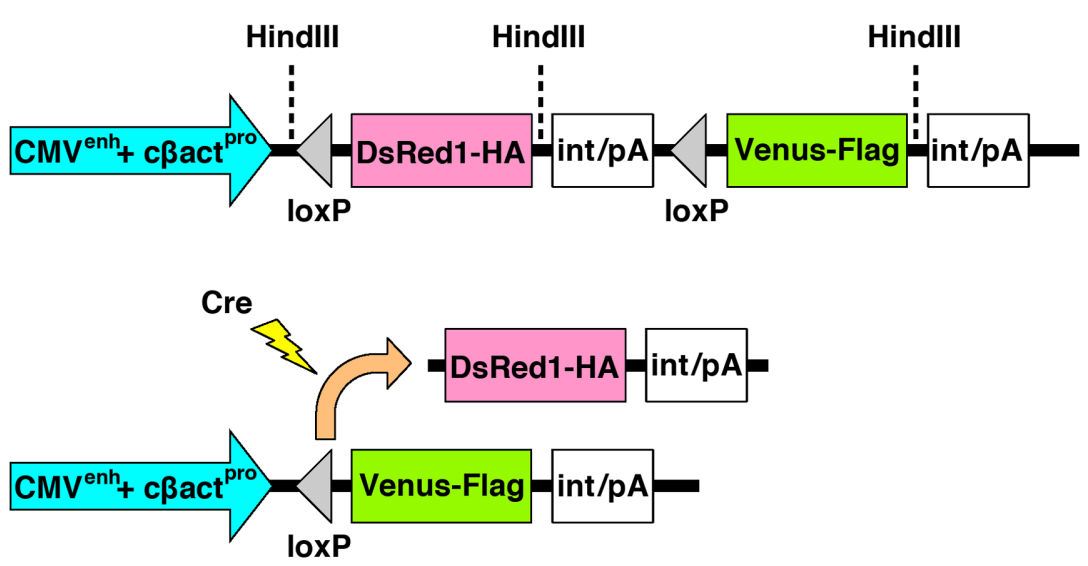

B

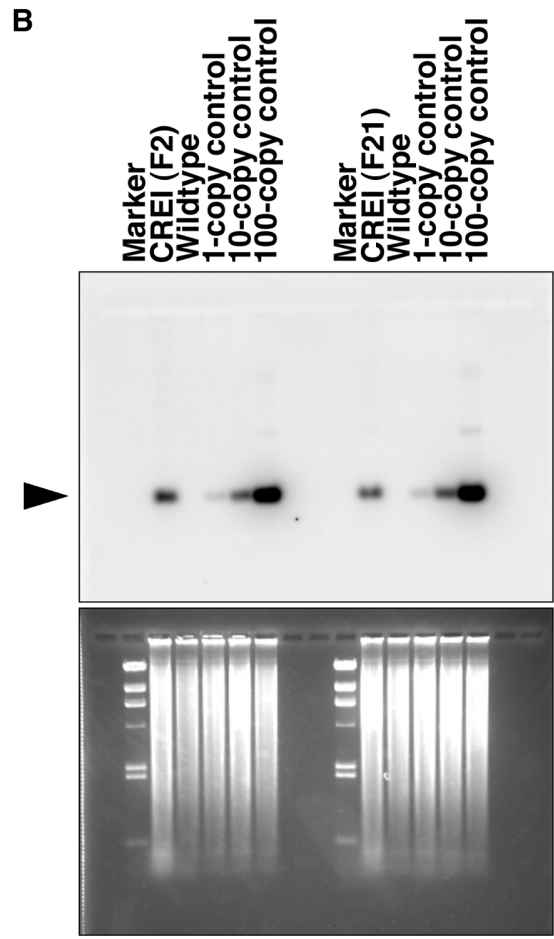

C

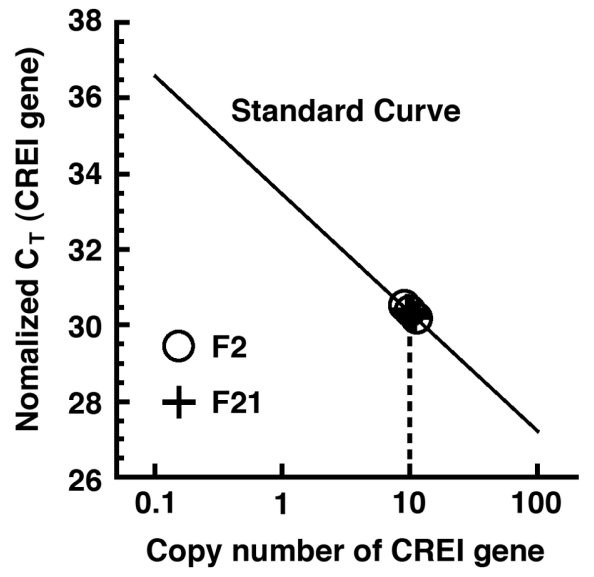

Fig. 1. (A) Schematic of the CREI gene. The CMV IE enhancer and the chick $\beta$-actin promoter are used to express constitutively and ubiquitously the DsRed1-HA gene or the Venus-Flag gene in mice. Cre recombinase changes the expressed reporter from DsRed1-HA to Venus-Flag by excision of the gene region between the two loxP sites. The "int/pA" shows the intron and polyadenylation site region derived from the SV40 T antigen gene. HindIII is a restriction enzyme used for the Southern blot analysis described below. (B) Southern blot analysis of the transgene in CREI mice. The upper panel shows an autoradiographic comparison of the F2 generation and the F21 generation of CREI mice, and of CREI mice and copy-number controls. The arrowhead indicates expected signals. The lower panel shows an ethidium bromide (EtBr)-stained image as a DNA loading control. (C) Quantitative PCR analysis of the transgene in CREI mice. The analysis was performed by using genomic DNA isolated from the F2 and F21 generations of CREI mice ( $n=3)$. $\beta$-actin was used for normalization.

imaging system with a monochrome CCD camera that was much more sensitive in the detection of fluorescent signals. In this observation, Meox-Cre; CREI mice expectedly showed fluorescent signals of DsRed1-HA in both the embryo and the placenta, and fluorescent signals of Venus-Flag in the embryo only. On the other hand,
CREI mice also showed DsRed1-HA signals in both the embryo and the placenta, but showed Venus-Flag signals in neither the embryo nor the placenta. Wild-type mice and Meox-Cre mice did not show either reporter signal in the embryo or the placenta (Fig. 4B).

The use of the in vivo imaging system not only enabled 
A

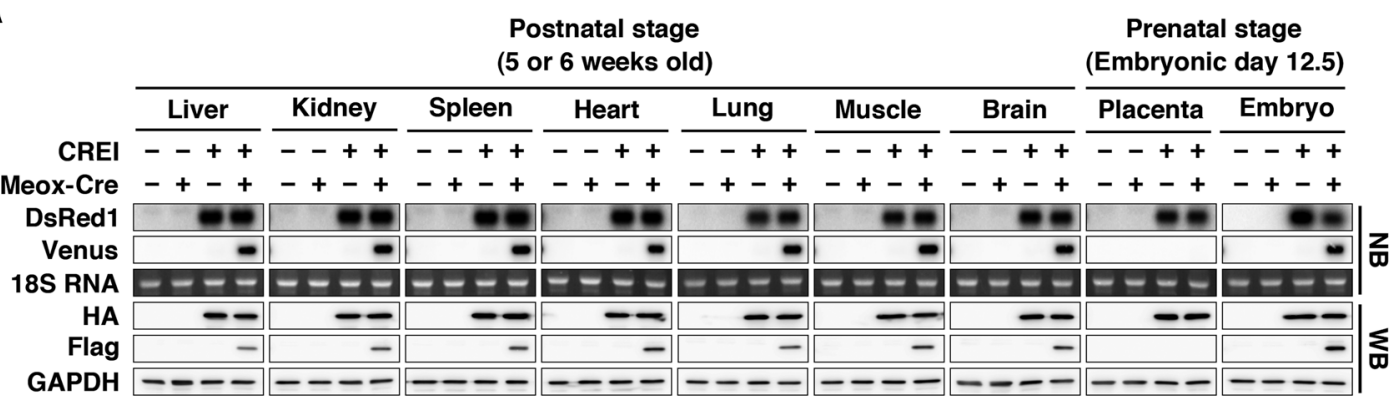

B

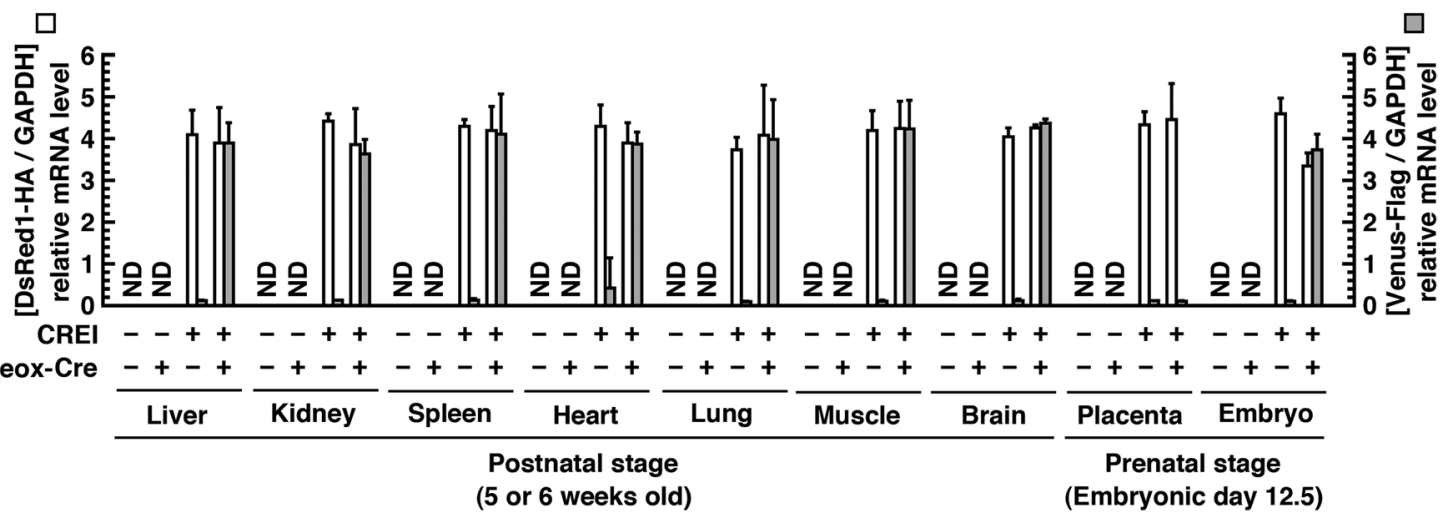

Fig. 2. (A) Northern blot and Western blot analysis of CREI reporters in mouse tissues. In the Northern blot analysis (NB), the upper and middle panels show the DsRed1-HA and Venus-Flag signals, respectively. The lower panel shows an EtBr-stained image of $18 \mathrm{~S}$ rRNA as a loading control. In the Western blot analysis (WB), the upper and middle panels show the DsRed1-HA and VenusFlag signals, respectively. The lower panel shows signals of GAPDH as a loading control. (B) Quantitative PCR analysis of CREI reporters in mouse tissues. The open and hatched columns show expression levels of DsRed1-HA mRNA and Venus-Flag mRNA, respectively. The expression level of endogenous Gapdh mRNA was measured as an internal standard. This histogram is shown as the mean (column) \pm SEM (error bar) from triplicate experiments.
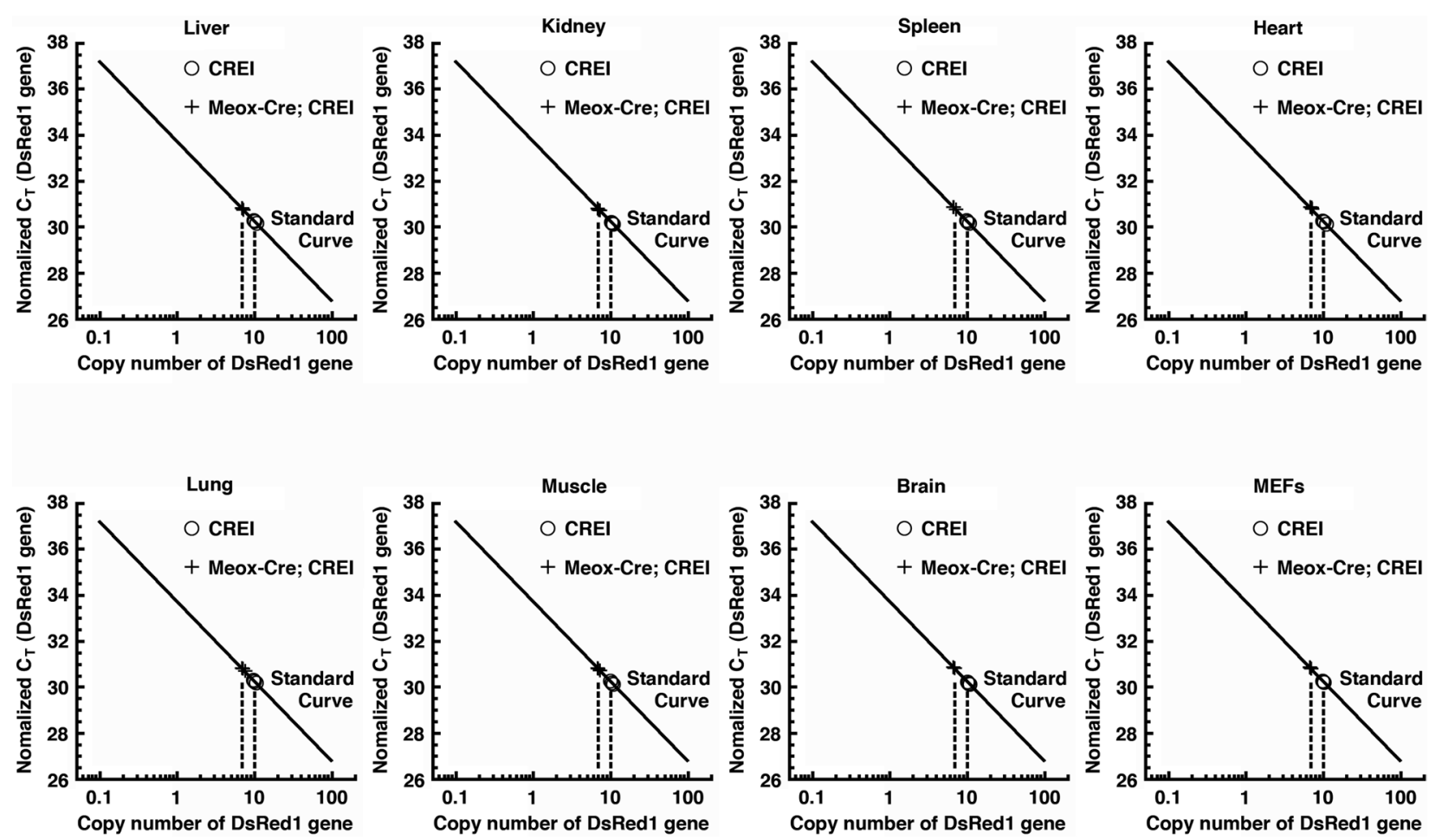

Fig. 3. The copy number of DsRed1-HA gene compared between in genome of CREI mice and Meox-Cre; CREI mice by quantitative PCR analysis. The analysis was performed by using genomic DNA isolated from the indicated tissues of 5 or 6-week-old mice $(n=3)$ and mouse embryonic fibroblasts (MEFs) of E12.5 embryos $(n=3)$. $\beta$-actin was used for normalization. 
A
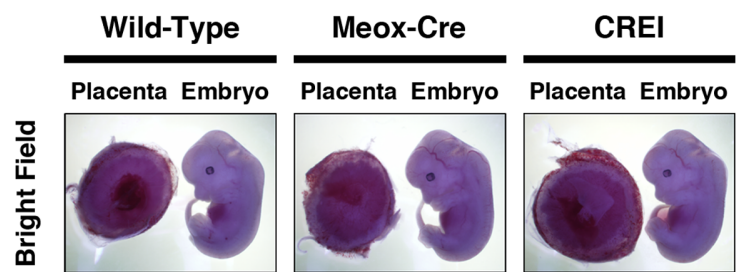

Meox-Cre; CREI
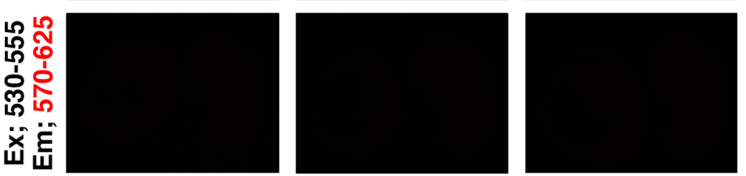

Placenta Embryo
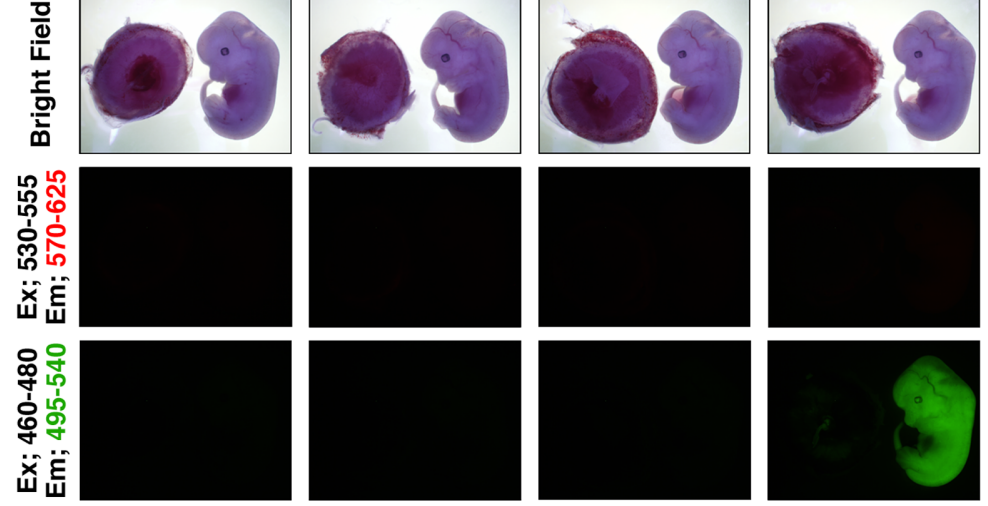

B

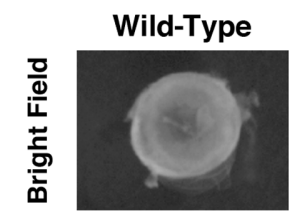

Meox-Cre
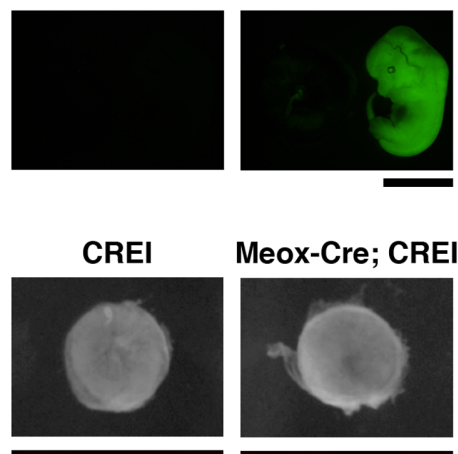

$\frac{\pi}{5}$
$\frac{0}{0}$
$\frac{\pi}{\alpha}$
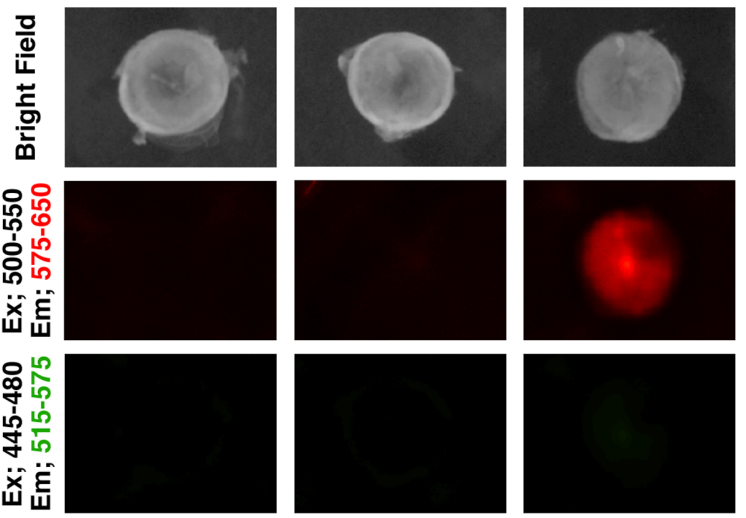

Meox-Cre; CREI
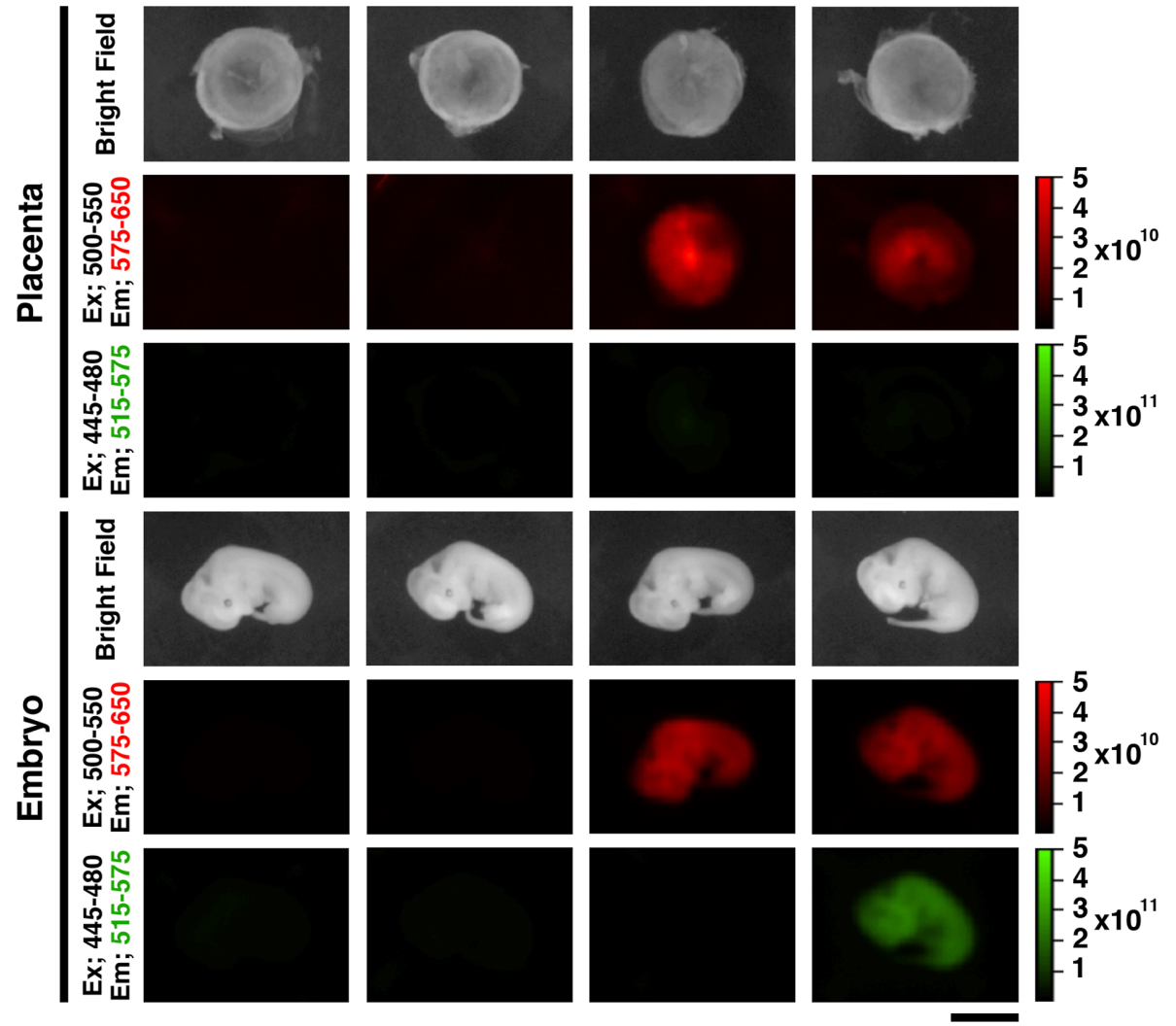

Fig. 4. (A) Images of mouse embryos and placentas observed with a fluorescent stereoscopic microscope. An excitation filter (530-555) and an emission filter (570-625) were used for observation of the DsRed1-HA signals. An excitation filter (460-480) and an emission filter (495-540) were used for observation of the Venus-Flag signals. The scale bar corresponds to $5 \mathrm{~mm}$. (B) Images of mouse embryos and placentas observed with an in vivo imaging system. An excitation filter (500-550) and an emission filter (575-650) were used for the observation of the DsRed1-HA signals. An excitation filter (445-480) and an emission filter (515-575) were used for the observation of the Venus-Flag signals. The scale bar corresponds to $5 \mathrm{~mm}$. The units for the signal indicators are $\mathrm{p} / \mathrm{s} / \mathrm{cm}^{2} / \mathrm{sr}$.

us to detect weak fluorescent signals, but also to measure the level of fluorescent signals in the regions of interest (Supplementary Fig. 1A, see Supplementary Information). This measurement of the fluorescent signals in the embryo and the placenta of each genotype mouse indicated that the strength of the DsRed1-HA signals was
$10-20 \%$ of that of the Venus-Flag signals (Supplementary Fig. 1B).

In addition to analysis in prenatal stage, we examined the fluorescent signals in postnatal stage of CREI mice. The fluorescent signals of DsRed1-HA and Venus-Flag from adult tissues showed the same pattern as those of the 
A

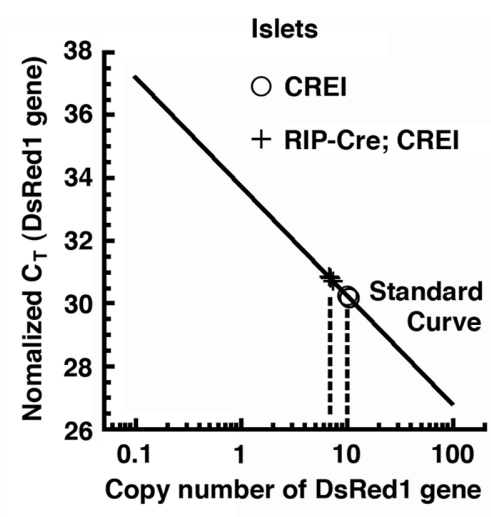

B

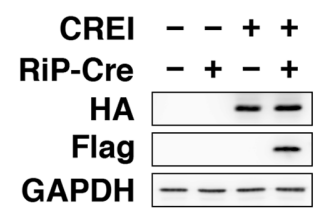

C
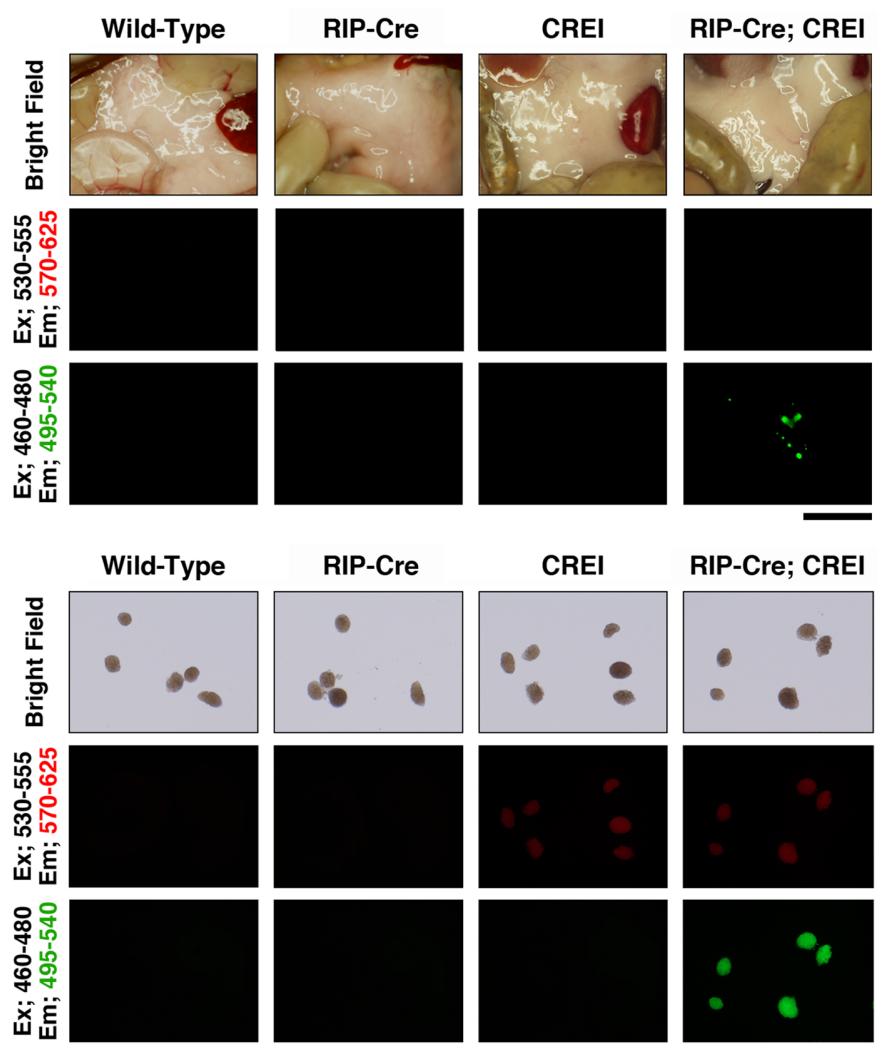

RIP-Cre; CREI

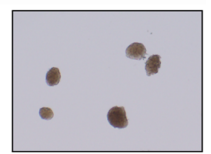

RIP-Cre; CREI
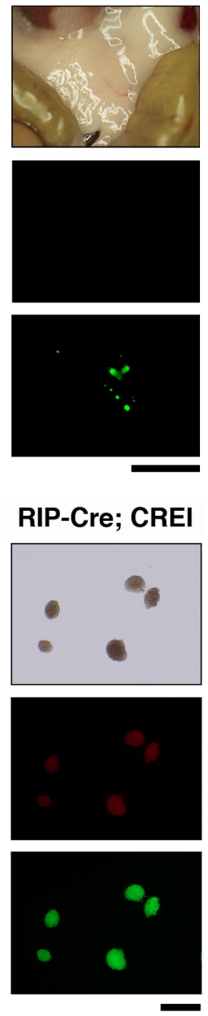

Fig. 5. (A) The copy number of DsRed1-HA gene compared between in genome of CREI mice and RIP-Cre; CREI mice by quantitative PCR analysis. The analysis was performed by using genomic DNA isolated from the islets of 8-week-old mice $(\mathrm{n}=3)$. $\beta$-actin was used for normalization. (B) Western blot analysis of CREI reporters in the islets. The upper and middle panels show the signals for DsRed1-HA and Venus-Flag, respectively. The lower panel shows signals of GAPDH as a loading control. (C) Images of the pancreas (upper panels) and the isolated islets (lower panels) observed with a fluorescent stereoscopic microscope. An excitation filter (530-555) and an emission filter (570-625) were used for observation of the DsRed1-HA signals. An excitation filter (460-480) and an emission filter (495-540) were used for observation of the VenusFlag signals. The upper and lower scale bars correspond to $2 \mathrm{~mm}$ and $200 \mu \mathrm{m}$, respectively.

embryos (Supplementary Figs. 2-9). The property of CREI mice was also analyzed by mating with RIP-Cre mice that specifically express Cre recombinase in $\beta$ cells of pancreatic islets. Quantitative PCR analysis of the isolated islets confirmed that CREI (RIP-Cre ${ }^{-}$) mice had ten copies of DsRed1-HA gene, but that RIP-Cre; CREI mice had 6-8 copies of DsRed1-HA gene (Fig. 5A). Western blot analysis of the isolated islets revealed that DsRed1HA protein was equivalently expressed in both of CREI (RIP-Cre ${ }^{-}$) mice and RIP-Cre; CREI mice, and that VenusFlag protein was expressed only in RIP-Cre; CREI mice (Fig. 5B). Observation with the fluorescence stereoscopic microscope and the color CCD camera showed that Venus-Flag signals were robustly detected from the islets of RIP-Cre; CREI mice, and that DsRed1-HA signals were marginally detected from the islets of CREI (RIP-Cre ${ }^{-}$) mice and RIP-Cre; CREI mice (Fig. 5C).

\section{Functional evaluation by using culture cells derived from CREI transgenic mice}

The results of the experiments performed in vivo, as described above, suggested that CREI mice might exhibit a weak red fluorescence before Cre/loxP-mediated recombination and a strong green fluorescence afterwards. Such a property might alleviate concern regarding errors in the judgment of signals and might increase the level of certainty in the detection of Cre activity, because any fluorescence signals in the green region of the spectrum leaking from the weak red fluorescence signals should be at too low level to permit their detection by a conventional fluorescence microscope. To investigate this hypothesis, we compared the reporter protein expression and fluorescent activity of MEFs derived from the CREI mouse with those of NIH3T3 cells transfected with the CREI gene (Fig. 6).

Both $\mathrm{MEFs}^{\mathrm{Cre}(-) / \mathrm{CREI}(+)}$ and $\mathrm{MEFs}^{\mathrm{Cre}(+) / \mathrm{CREI}(+)}$ expressed DsRed1-HA proteins and showed red fluorescent signals at a low level. Those signals were detected under long-exposure conditions but not under short-exposure conditions. MEFs ${ }^{\mathrm{Cre}(+) / C R E I(+)}$ also expressed Venus-Flag proteins and showed green fluorescent signals at a high level. The signals were detected under short-exposure 

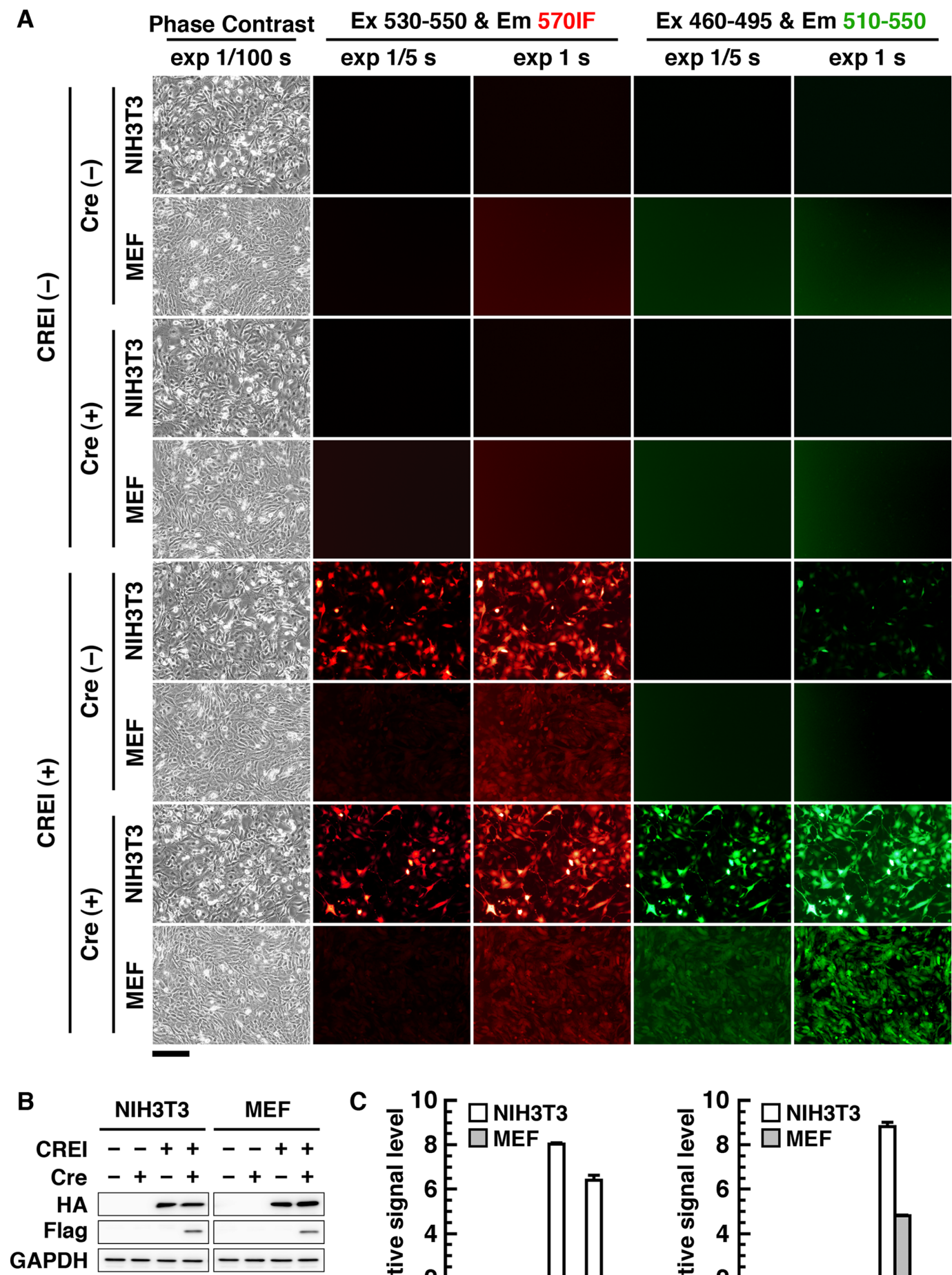

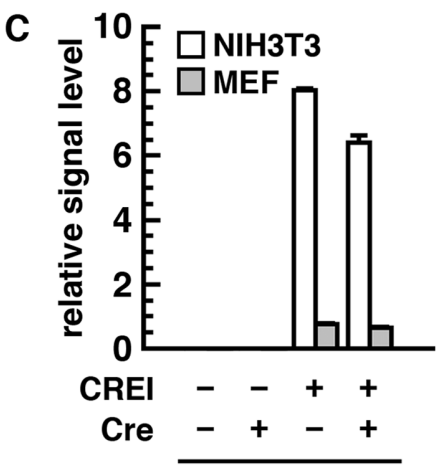

Ex 544/15 \& Em 590/14

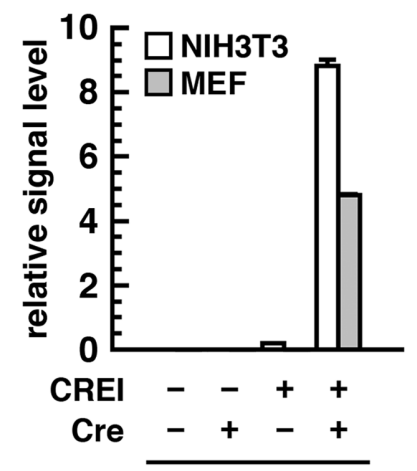

Ex 485/14 \& Em 538/25

Fig. 6. (A) Images of cultured cells observed with a fluorescent microscope. An excitation filter (530-555) and an emission filter (570IF) were used to observe the DsRed1-HA signals. An excitation filter (460-495) and an emission filter (510-550) were used to observe the Venus-Flag signals. The scale bar corresponds to $200 \mu \mathrm{m}$. (B) Western blot analysis of CREI reporters in culture cells. The upper and middle panels show the signals for DsRed1-HA and Venus-Flag, respectively. The lower panel shows signals of GAPDH as a loading control. (C) Measurement of fluorescent signals from cultured cells. The open and hatched columns show fluorescent signal levels of the NIH3T3 cells and mouse embryonic fibroblasts (MEFs), respectively. An excitation filter (544/15) and an emission filter (590/14) were used in measuring the DsRed1-HA signals. An excitation filter (485/14) and an emission filter (538/25) were used in measuring the Venus-Flag signals. These histograms are shown as the mean (column) \pm SEM (error bar) from triplicate experiments. 


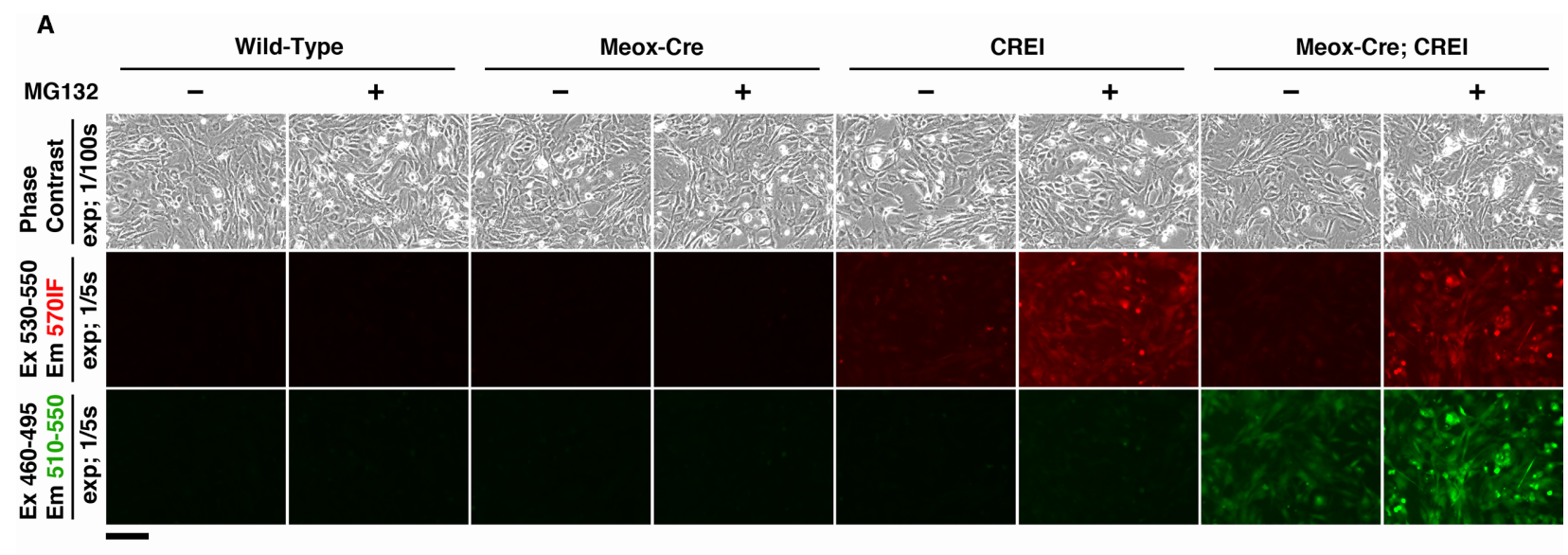

B

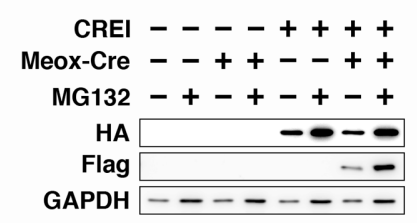

C

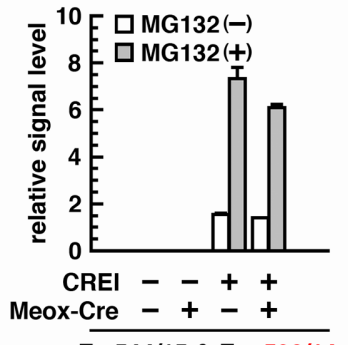

Ex 544/15 \& Em 590/14

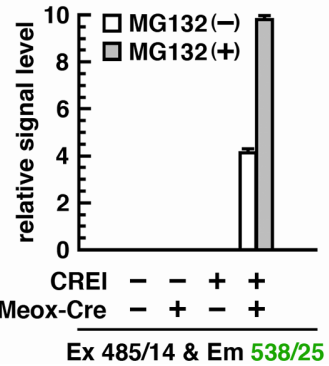

Fig. 7. (A) Fluorescent microscopic images of mouse embryonic fibroblasts (MEFs) treated without or with MG132. An excitation filter (530-555) and an emission filter (570IF) were used to observe the DsRed1-HA signals. An excitation filter (460-495) and an emission filter (510-550) were used to observe the Venus-Flag signals. The scale bar corresponds to $200 \mu \mathrm{m}$. (B) Western blot analysis of CREI reporters in MEFs. The upper and middle panels show the signals for DsRed1-HA and Venus-Flag, respectively. The lower panel shows signals of GAPDH as a loading control. (C) Measurement of fluorescent signals from MEFs. The open or hatched columns show fluorescent signal levels of MEFs treated without or with MG132, respectively. An excitation filter (544/15) and an emission filter (590/14) were used in measuring the DsRed1-HA signals. An excitation filter (485/14) and an emission filter (538/25) were used in measuring the Venus-Flag signals. These histograms are shown as the mean (column) \pm SEM (error bar) from triplicate experiments.

conditions as well as under long-exposure conditions. $\mathrm{MEFs}^{\mathrm{Cre}(-) / \mathrm{CREI}(+)}$ did not express Venus-Flag proteins and did not show any detectable green fluorescence, even under long-exposure conditions. On the other hand, both $\mathrm{NIH} 3 \mathrm{~T}^{\mathrm{Cre}(-) / \mathrm{CREI}(+)}$ cells and NIH3T3 ${ }^{\mathrm{Cre}(+) / \mathrm{CREI}(+)}$ cells expressed DsRed1-HA proteins and produced much stronger red fluorescent signals that could be detected under short-exposure conditions as well as under longexposure conditions. $\mathrm{NIH} 3 \mathrm{~T}^{\mathrm{Cre}(+) / C R E I(+)}$ cells also expressed Venus-Flag proteins and showed a much stronger green fluorescence that could be detected under both short-exposure and long-exposure conditions. NIH3T$3^{\mathrm{Cre}(-) / \mathrm{CREI}(+)}$ cells did not express Venus-Flag proteins, but showed a much weaker green fluorescence that could be marginally detected under long-exposure conditions, but not under short-exposure conditions.

To address the reason why DsRed1-HA signals are weak in cells and tissues derived from CREI mice, we next evaluated fluorescent signals and protein levels of MEFs treated with MG132, which is known as a proteasome inhibitor. Venus-Flag signals were increased up to 2.2 times in $\mathrm{MEFs}{ }^{\mathrm{Cre}(+) / \mathrm{CREI}(+)}$ by treatment with
MG132. On the other hand, DsRed1-HA signals were increased up to 3-4 times in $\mathrm{MEFs}{ }^{\mathrm{Cre}(-) / \mathrm{CREI}(+)}$ and $\mathrm{MEF}$ $\mathrm{s}^{\mathrm{Cre}(+) / \mathrm{CREI}(+)}$ by treatment with it. Increase of protein levels by treatment with MG132 was more prominent in DsRed1-HA than in endogenous GAPDH (Fig. 7).

These MG132-induced effects also facilitate flow cytometry analysis of MEFs derived from CREI mice. Flow cytometry analysis of MEFs treated with MG132 also revealed that $1.0 \%$ of $\mathrm{MEFs}^{\mathrm{Cre}(-) / \mathrm{CREI}(+)}$ and $63.7 \%$ of $\mathrm{MEFs}^{\mathrm{Cre}(+) / \mathrm{CREI}(+)}$ were double positive of DsRed 1 and Venus, that $36.1 \%$ of $\mathrm{MEFs}^{\mathrm{Cre}(-) / \mathrm{CREI}(+)}$ and $30.4 \%$ of $\mathrm{MEFs}^{\mathrm{Cre}(+) / \mathrm{CREI}(+)}$ were double negative of DsRed 1 and Venus, and that $62.9 \%$ of MEFs ${ }^{\mathrm{Cre}(-) / \mathrm{CREI}(+)}$ and $5.6 \%$ of $\mathrm{MEFs}^{\mathrm{Cre}(+) / \mathrm{CREI}(+)}$ were DsRed $1^{+}$and Venus ${ }^{-}$(Fig. 8). However, immunohistological analysis of embryonic liver with anti-RFP antibody and anti-GFP antibody showed that DsRed $1^{+} /$Venus $^{-}$cells and DsRed $1^{+} /$Venus $^{+}$ cells predominantly occupied tissue sections of CREI $\left(\right.$ Meox-Cre $\left.{ }^{-}\right)$mice and Meox-Cre; CREI mice, respectively (Fig. 9). 

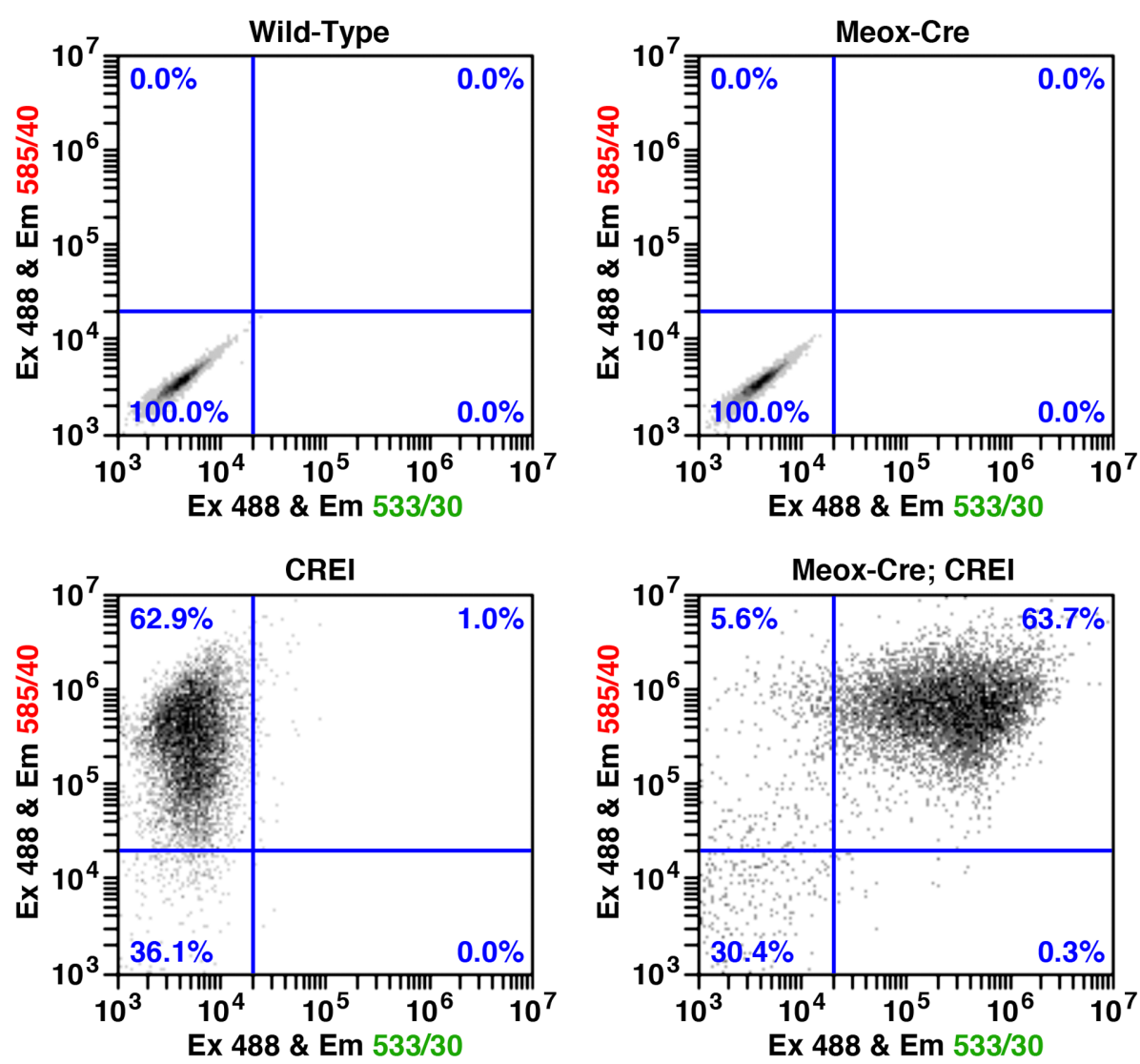

Fig. 8. Flow cytometry analysis of MG132-treated mouse embryonic fibroblasts (MEFs). An excitation laser (488) and an emission filter (585/40) were used to detect the DsRed1-HA signals. An excitation laser (488) and an emission filter (533/30) were used to detect the Venus-Flag signals. Each chart shows the same plot number (10,000 events). Data of WildType MEFs and Meox-Cre MEFs was used for gating (blue lines) of negative or positive fluorescent signals.
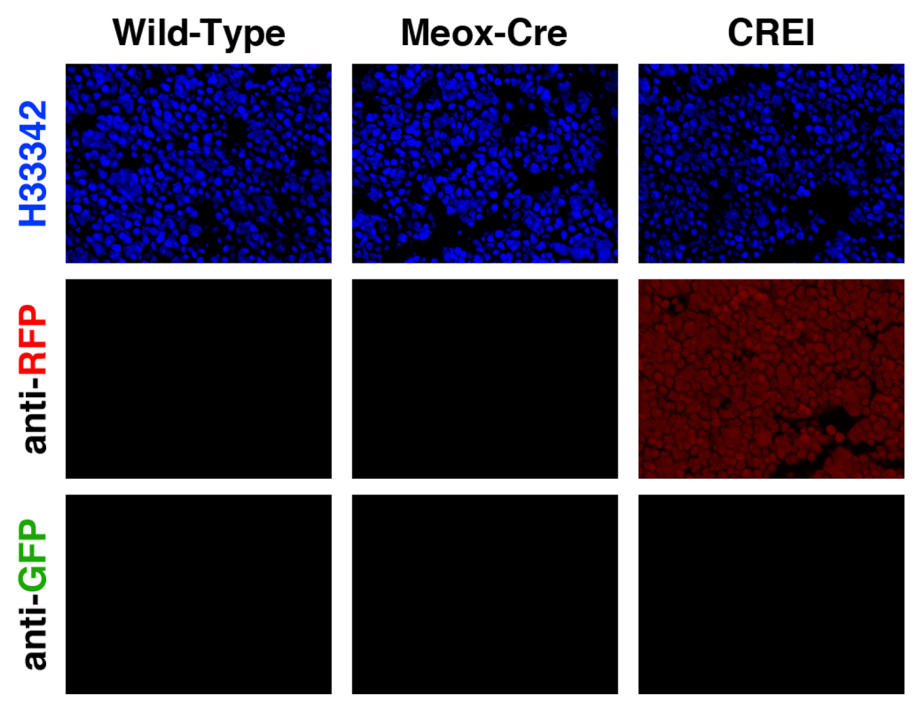

Meox-Cre; CREI
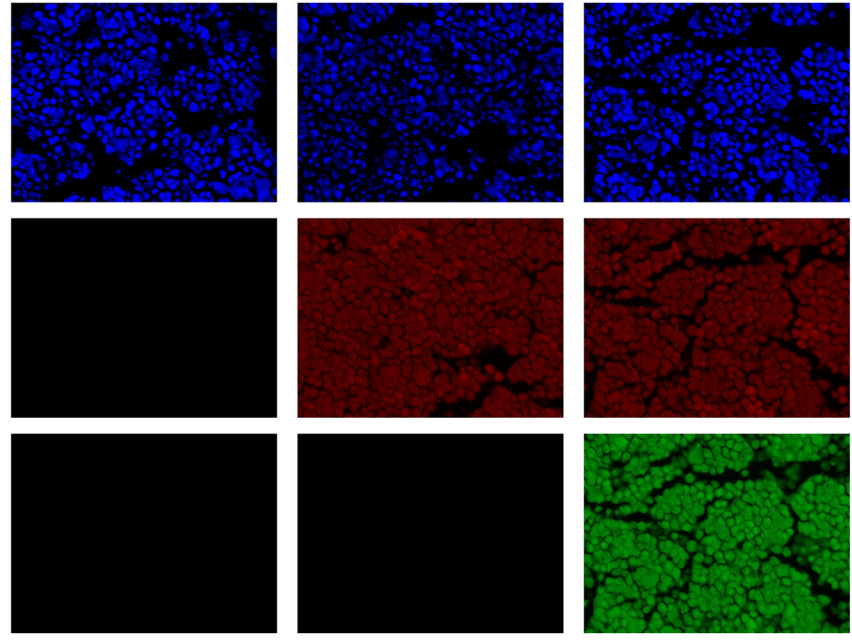

Fig. 9. Immunohistological images of tissue sections derived from embryonic liver. Anti-red fluorescent protein (RFP) antibody was used for observation of the DsRed1-HA signals. Anti-green fluorescent protein (GFP) antibody was used for observation of the Venus-Flag signals. Hoechst 33342 (H33342) was used for counter staining of the tissues. The scale bar corresponds to $50 \mu \mathrm{m}$. 


\section{Discussion}

In the present research, we fortuitously developed a transgenic mouse (CREI) line that is valuable for monitoring the activity of Cre recombinase in vivo. The CREI mice expectedly expressed DsRed1-HA under Crenegative conditions, and expressed Venus-Flag under Cre-positive conditions (Fig. 2). Consequently, we could easily detect Cre activities in vivo from the green fluorescent signals produced by the CREI mice (Fig. 4 and Supplementary Figs. 2-9). Other mouse lines have already been reported to play similar roles as Cre indicators $[4,5]$. However, our CREI mouse line is different from the other mouse lines in that the reporter signals are emitted at relatively low levels under Cre-negative conditions. This difference provides the CREI mouse line with a beneficial feature. This is because strong DsRed1 signals are liable to show some fluorescence emission in the green region of the spectrum, whereas weak DsRed1 signals do not so (Fig. 6). Therefore CREI mice are less likely to show false green fluorescent signals, and are useful for the exclusive detection of Venusderived green fluorescent signals.

As far as we examined, all tissues and cells derived from CREI mice expressed DsRed1-HA at an adequate level, but showed weak red fluorescent signals. This feature is probably attributed to unstable property of DsRed1 protein. It was reported that DsRed1 is stable in dimeric state, but is rapidly degraded in monomeric state through the ubiquitin-proteasome pathway [9]. In fact, our experiment by using MG132 revealed that CREI MEFs were able to show increased red fluorescent signals and high expression level of DsRed1-HA protein (Fig. 7).

CREI mice expressed DsRed1-HA and showed weak red fluorescence, not only under Cre-negative conditions, but also under Cre-positive conditions (Figs. 2, 4 and Supplementary Figs. 2-9). Flow cytometry analysis and Immunohistological analysis indicated that DsRed $1^{+} /$ Venus $^{+}$cells predominantly occupied Meox-Cre; CREI mice (Figs. 8 and 9). Comparison of the copy number of DsRed1-HA gene between in genome of CREI (Meox$\mathrm{Cre}^{-}$) mice and in that of Meox-Cre; CREI mice suggests that $6-8$ copies of transgene might be unresponsive to Cre recombinase in every tissue and in MEFs (Fig. 3). Similar unresponsiveness to Cre recombinase was also showed in the islets of RIP-Cre; CREI mice (Fig. 5A). Therefore we speculate that this partial reactivity of CREI transgene is dependent on some specific property of CREI mice, not dependent on that of Meox-Cre mice and RIP-Cre mice, and causes expression of DsRed1-HA under Cre-positive conditions.
Fluorescent signals shown in the presented CREI mouse line turned from weak $\underline{R} F P$ / no GFP to weak RFP / strong GFP in Cre dependent manner. From this property we named the presented mouse line as "CREI WRtoSG" to separate it from other Cre indicator mouse lines. This name will be used for future reference. As described above, long-exposure conditions and/or higher sensitive CCD camera are required for detection of the weak RFP signals from CREI ${ }^{\text {WRtoSG }}$ mice. The weak RFP signals are also showed in CREI ${ }^{\mathrm{WRt} t o S G}$ mice even under Crepositive conditions. These may be considered as defective properties in Cre indicator mice. However, the Credependent GFP signals could be easily detected without concern for leak signals derived from RFP by using our CREI ${ }^{\text {WRtoSG }}$ mice. Considering that Cre-dependent reporter activity is generally critical property in Cre indicator mice, our CREI ${ }^{\text {WRtoSG }}$ mice provide satisfactory performance as a Cre indicator. If we had generated a contrary transgenic mouse model exhibiting strong green fluorescence before and weak red fluorescence after Cre recombination, it would have provided unsatisfactory performance as a Cre indicator for interfering effect of strong green fluorescence on weak red fluorescence.

Regarding other critical properties as a Cre indicator, we confirmed that CREI ${ }^{\text {WRtoSG }}$ mice ubiquitously express the reporter gene (Fig. 2), and that $\mathrm{CREI}^{\mathrm{WRtoSG}}$ mice are useful for analysis by mating with two kinds of Cre-expressing (Meox-Cre and RIP-Cre) mice (Supplementary Figs. 2-9 and Fig. 5). Recently, many researchers have intensively analyzed conditional gene knockout mice by using the Cre/loxP system. From these beneficial aspects and increasing needs, we concluded that CREI ${ }^{\text {WRtoSG }}$ mouse could become an indispensable model tool for the performance of such animal experiments.

\section{Conflict of Interest}

The authors declare no competing or financial interests.

\section{Acknowledgments}

We thank Hisayo Hamashima and Hideaki Ninomiya for technical assistance, and we are grateful to the Research Support Center (Medical Research Institute, Kanazawa Medical University) for breeding the mice and for the use of their experimental equipment. This work was supported by grants from Kanazawa Medical University (to R.A. and T.I.), the Research Foundation for Opto-Science and Technology (to T.I.), the Nakatani Foundation (to T.I.), the Toray Science Foundation (to T.I.), the Takeda Science Foundation (to K.K.), and MEXT (to T.I. and K.K.). 


\section{References}

1. Akagi, K., Sandig, V., Vooijs, M., Van der Valk, M., Giovannini, M., Strauss, M. and Berns, A. 1997. Cre-mediated somatic site-specific recombination in mice. Nucleic Acids Res. 25: 1766-1773. [Medline] [CrossRef]

2. Burn, S.F. 2012. Detection of $\beta$-galactosidase activity: Xgal staining. Methods Mol. Biol. 886: 241-250. [Medline] [CrossRef]

3. Guo, F., Gopaul, D.N. and van Duyne, G.D. 1997. Structure of Cre recombinase complexed with DNA in a site-specific recombination synapse. Nature 389: 40-46. [Medline] [CrossRef]

4. Hartwich, H., Satheesh, S.V. and Nothwang, H.G. 2012. A pink mouse reports the switch from red to green fluorescence upon Cre-mediated recombination. BMC Res. Notes 5: 296. [Medline] [CrossRef]

5. Hasegawa, Y., Daitoku, Y., Sekiguchi, K., Tanimoto, Y., Mizuno-Iijima, S., Mizuno, S., Kajiwara, N., Ema, M., Miwa, Y., Mekada, K., Yoshiki, A., Takahashi, S., Sugiyama, F. and Yagami, K. 2013. Novel ROSA26 Cre-reporter knock-in C57BL/6N mice exhibiting green emission before and red emission after Cre-mediated recombination. Exp. Anim. 62: 295-304. [Medline] [CrossRef]

6. Hoess, R.H. and Abremski, K. 1984. Interaction of the bacteriophage $\mathrm{P} 1$ recombinase Cre with the recombining site loxP. Proc. Natl. Acad. Sci. USA 81: 1026-1029. [Medline] [CrossRef]

7. Hoffman, R.M. 2008. Imaging In Mice With Fluorescent Proteins: From Macro To Subcellular. Sensors (Basel) 8: 11571173. [Medline] [CrossRef]

8. Iwawaki, T., Akai, R., Yamanaka, S. and Kohno, K. 2009. Function of IRE1 alpha in the placenta is essential for placental development and embryonic viability. Proc. Natl. Acad. Sci. USA 106: 16657-16662. [Medline] [CrossRef]

9. Kagoshima, H., Nimmo, R., Saad, N., Tanaka, J., Miwa, Y., Mitani, S., Kohara, Y. and Woollard, A. 2007. The C. elegans CBFbeta homologue BRO-1 interacts with the Runx factor, RNT-1, to promote stem cell proliferation and self-renewal. Development 134: 3905-3915. [Medline] [CrossRef]

10. Lewandoski, M. 2001. Conditional control of gene expression in the mouse. Nat. Rev. Genet. 2: 743-755. [Medline] [CrossRef]

11. Li, D.S., Yuan, Y.H., Tu, H.J., Liang, Q.L. and Dai, L.J. 2009. A protocol for islet isolation from mouse pancreas. Nat. Pro- toc. 4: 1649-1652. [Medline] [CrossRef]

12. Magnuson, M.A. and Osipovich, A.B. 2013. Pancreas-specific Cre driver lines and considerations for their prudent use. Cell Metab. 18: 9-20. [Medline] [CrossRef]

13. Matz, M.V., Fradkov, A.F., Labas, Y.A., Savitsky, A.P., Zaraisky, A.G., Markelov, M.L. and Lukyanov, S.A. 1999. Fluorescent proteins from nonbioluminescent Anthozoa species. Nat. Biotechnol. 17: 969-973. [Medline] [CrossRef]

14. Miyawaki, A., Sawano, A. and Kogure, T. 2003. Lighting up cells: labelling proteins with fluorophores. Nat. Cell Biol. (Suppl): S1-S7. [Medline]

15. Nagai, T., Ibata, K., Park, E.S., Kubota, M., Mikoshiba, K. and Miyawaki, A. 2002. A variant of yellow fluorescent protein with fast and efficient maturation for cell-biological applications. Nat. Biotechnol. 20: 87-90. [Medline] [CrossRef]

16. Nagy, A. 2000. Cre recombinase: the universal reagent for genome tailoring. Genesis 26: 99-109. [Medline] [CrossRef]

17. Nagy, A., Gertsenstein, M., Vintersten, K. and Behringer, R. 2003. Manipulating the Mouse Embryo: A Laboratory Manual, Third Edition. Cold Spring Harbor Laboratory Press, New York.

18. Sauer, B. 1987. Functional expression of the cre-lox site-specific recombination system in the yeast Saccharomyces cerevisiae. Mol. Cell. Biol. 7: 2087-2096. [Medline] [CrossRef]

19. Sauer, B. and Henderson, N. 1988. Site-specific DNA recombination in mammalian cells by the Cre recombinase of bacteriophage P1. Proc. Natl. Acad. Sci. USA 85: 5166-5170. [Medline] [CrossRef]

20. Shao, M., Shan, B., Liu, Y., Deng, Y., Yan, C., Wu, Y., Mao, T., Qiu, Y., Zhou, Y., Jiang, S., Jia, W., Li, J., Li, J., Rui, L., Yang, L. and Liu, Y. 2014. Hepatic IRE1 $\alpha$ regulates fasting-induced metabolic adaptive programs through the XBP1s-PPAR $\alpha$ axis signalling. Nat. Commun. 5: 3528. [Medline] [CrossRef]

21. Soriano, P. 1999. Generalized lacZ expression with the ROSA26 Cre reporter strain. Nat. Genet. 21: 70-71. [Medline] [CrossRef]

22. Tallquist, M.D. and Soriano, P. 2000. Epiblast-restricted Cre expression in MORE mice: a tool to distinguish embryonic vs. extra-embryonic gene function. Genesis 26: 113-115. [Medline] [CrossRef]

23. Zhang, K., Wang, S., Malhotra, J., Hassler, J.R., Back, S.H., Wang, G., Chang, L., Xu, W., Miao, H., Leonardi, R., Chen, Y.E., Jackowski, S. and Kaufman, R.J. 2011. The unfolded protein response transducer IRE1 $\alpha$ prevents ER stress-induced hepatic steatosis. EMBO J. 30: 1357-1375. [Medline] [CrossRef] 